\title{
Impact of Wireless-Powered Communications in Coexisting Mobile Networks
}

\author{
Luis Irio, Rodolfo Oliveira, Senior Member, IEEE, Daniel B. da Costa, Senior \\ Member, IEEE, and Mohamed-Slim Alouini, Fellow, IEEE
}

\begin{abstract}
In this letter, we investigate the impact of wireless-powered communications when energy is harvested from multiple static and/or mobile wireless coexisting networks. In a first step, we characterize the aggregate power received by a harvester node when it harnesses the energy generated by the coexisting wireless networks. Considering that the harvester node acts as a transmitter after the harvesting duration, we derive the outage probability for such coexisting scenario. In addition, the throughput achieved by the harvester node is also characterized, and the optimal harvesting duration is identified taking into account the mobility of the coexisting networks, the features of the static networks, the energy harvesting process, as well as the communication performance between the harvester node and the receiver. Our work shows that the distribution of the power received by the harvester from the coexisting networks can be accurately approximated by an $\alpha-\mu$ distribution. Moreover, the mobility also impacts on the optimal throughput of the wireless-powered communications, which is accurately confirmed by the proposed analysis and extensive simulations.
\end{abstract}

\section{Index Terms}

mobility, coexistence, energy-harvesting, performance evaluation.

\section{INTRODUCTION}

Wireless-powered communications (WPC) have been recently proposed to extend the network's lifetime. In WPCs, the aggregate energy collected by a harvester node from multiple radio-frequency $(\mathrm{RF})$ signals is used to power the communication process. Coexisting interference

L. Irio, and R. Oliveira are with the Instituto de Telecomunicações, 1049-001 Lisbon, Portugal, and also with the Departamento de Engenharia Electrotécnica, Faculdade de Ciências e Tecnologia, Universidade Nova de Lisboa, 2829-516 Caparica, Portugal (e-mail: 1.irio@campus.fct.unl.pt, rado@fct.unl.pt). D. B. da Costa is with the Department of Computer Engineering, Federal University of Ceará, Sobral-CE, Brazil (e-mail: danielbcosta@ieee.org). Mohamed-Slim Alouini is with the King Abdullah University of Science and Technology (KAUST), Thuwal, Saudi Arabia (e-mail: slim.alouini@kaust.edu.sa). 
was recently studied in [1] for static networks. The effect of the interference on the performance of energy harvesting systems has been analyzed in [2] and [3]. The interference caused by multiple transmitters was characterized in [4], which considers that different ambient RF energy sources cause interference to a wireless sensor node. The RF energy sources are distributed as a Ginibre $\alpha$-determinantal point process (DPP), and the performance analysis is stated as a stochastic geometry problem. Most of the existing works do not address large scale WPC networks due to the challenges associated with the characterization of the harvested RF energy in the presence of dominant transmitters. An exception to this is the work in [5], which uses the probability generating functional of a Poisson point process (PPP), to characterize the distribution of the harvested energy.

To the best of the authors' knowledge, the characterization of WPC systems that harvest energy from multiple coexisting networks (including static and/or mobile ones), has not yet been addressed in the literature. In order to fill partly this gap that exists in the literature, this work aims to first study the distribution of the harvested energy from multiple energy sources belonging to different coexisting networks. We consider the case where mobile and/or static networks may coexist together in the same band. Admitting that the harvester node acts as a transmitter after the energy harvesting period, we derive the outage probability for such scenario. In addition, we study the throughput achieved by the harvester node, identifying the optimal energy harvesting time allocation having into account the mobility of the mobile networks, the features of the static networks, the energy harvesting process, as well as the communication performance between the harvester node and the receiver.

The remainder of this paper is organized as follows. Section II introduces the system model adopted in the work. Section III characterizes the energy harvested by a node from different coexisting wireless networks, and Section IV describes the throughput achieved by the harvester node. Finally, Section V assesses the accuracy of the proposed methodology along with insightful discussions.

Notations and functions: $f_{X}($.$) and \mathrm{P}(X=x)$ represent the probability density function (PDF) and the probability of a random variable (RV) $X$, respectively. $\Gamma($.$) represents the complete$ Gamma function [6, Eq. (8.310.1)]. $\Gamma(.,$.$) denotes the incomplete Gamma function [6, Eq.$ (8.350.1)]. Nakagami $\left(x_{1}, x_{2}\right)$ denotes the Nakagami distribution with shape $x_{1}$ and spread $x_{2}$. $\mathrm{E}[X]$ and $\operatorname{Var}[X]$ are the expectation and variance of the $\mathrm{RV} X$, respectively. 


\section{System Model}

\section{A. Coexisting Wireless Networks}

In this work, we consider the scenario illustrated in Fig. 1, where multiple $\Upsilon_{\eta}$ networks, with $\eta=1, \ldots, v$, coexist in the same RF band, and same spatial region with area $X_{\max } \times Y_{\max }$. The $v$ networks can be static or mobile. The nodes of the static networks are deployed according to a homogeneous PPP. The nodes of the mobile networks move according to the Random Waypoint (RWP) mobility model [7], where each node is initially placed in a random position $(x, y)$ sampled from the uniform distributions represented by $x \in\left[0, X_{\max }\right]$ and $y \in\left[0, Y_{\max }\right]$, and move to a random ending point with velocity uniformly sampled from $\left[V_{\min }, V_{\max }\right]$. The nodes stop at the ending point for pause time $T_{p}$. After reaching the ending point a node repeats the same cycle. The average velocity of the nodes is denoted by $\mathrm{E}[V]$, which was studied in [7].

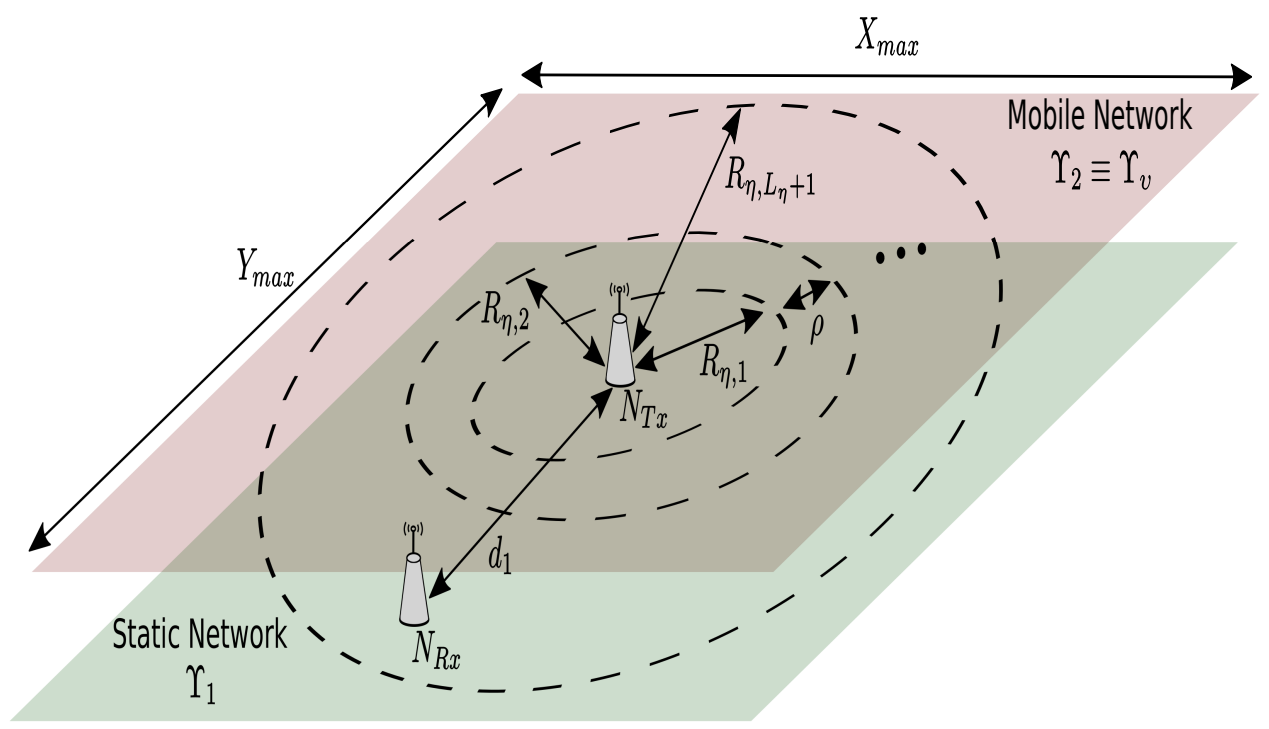

Fig. 1. A harvester node $N_{T x}$ receives energy from $v$ coexistent networks to transmit information to the node $N_{R x}$. The dashed circles represent the boundaries of the annuli considered in the proposed model. The inner circle radius is denoted by $R_{\eta, 1}$, and $R_{\eta, L_{\eta}+1}$ denotes the outer circle radius.

For modeling purposes, we adopt the spatial circular model (SCM) considered in [8], where the analysis of the energy received by the harvester node (node $N_{T x}$ in Fig. 1) from the network $\Upsilon_{\eta}$ is derived by considering the nodes located in the $L_{\eta}$ annuli centered on the harvester node. The radius of the larger and smaller circles of the annulus $l \in\left\{1, \ldots, L_{\eta}\right\}$, are represented by $R_{\eta, l+1}=\left(R_{\eta, l}+l \rho\right)$ and $R_{\eta, l}$, respectively, where $\rho$ denotes the annulus' width. The nodes 
of a given network $\Upsilon_{\eta}$ are thus located in a circular region with area $A_{\eta}=\sum_{l=1}^{L_{\eta}} A_{\eta, l}$, where $A_{\eta, l}=\pi\left(\left(R_{\eta, l+1}\right)^{2}-\left(R_{\eta, l}\right)^{2}\right)$ denotes the area of the annulus $l$.

The number of transmitters of the network $\Upsilon_{\eta}$ located in a particular annulus $l \in\left\{1, \ldots, L_{\eta}\right\}$ is represented by the $\mathrm{RV} X_{\eta, l}$. For both static and mobile networks we consider that $X_{\eta, l}$ is distributed according to a truncated Poisson distribution given by

$$
\mathrm{P}\left(X_{\eta, l}=k\right)=\frac{\left(\lambda_{\eta, l} A_{\eta, l} \tau_{\eta}\right)^{k} e^{-\lambda_{\eta, l} A_{\eta, l} \tau_{\eta}}}{k ! \sum_{i=0}^{n_{\eta}} \frac{\left(\lambda_{\eta, l} A_{\eta, l} \tau_{\eta}\right)^{i}}{i !} e^{-\lambda_{\eta, l} A_{\eta, l} \tau_{\eta}}}, 0 \leq k \leq n_{\eta},
$$

where $\lambda_{\eta, l}$ is the node's spatial density, $n_{\eta}$ is the total number of nodes of the network $\Upsilon_{\eta}$, and $\tau_{\eta}$ is the individual transmission probability. We highlight that for static networks $\lambda_{\eta, l}$ is equal for all $L_{\eta}$ annulus. However, for RWP mobile networks, the spatial distribution of the nodes is approximated by an inhomogeneous PPP. Consequently, for mobile RWP networks, $\lambda_{\eta, l}$ takes a different value for each annulus $l$. In this work, we consider that the density parameter $\lambda_{\eta, l}$ adopted to model the mobile RWP networks is computed as proposed in [9, Eq. (8)], which takes into account the annuli's geometry $\left(\rho ; L_{\eta} ; R_{\eta, 1}\right)$, and mobility parameters $\left(V_{\text {min }} ; V_{\text {max }} ; X_{\text {max }} ; Y_{\text {max }} ; T_{p}\right)$.

\section{B. Propagation Effects}

The power received by the harvester node $\left(N_{T x}\right)$ from the $n_{\eta, l}$ transmitters of the network $\Upsilon_{\eta}$ located in the annulus $l$ is denoted by $I_{\eta, l}=\sum_{i=1}^{n_{\eta, l}} I_{\eta, l, i} . I_{\eta, l, i}$ represents the power received from the $i$-th transmitter, which can be written as follows

$$
I_{\eta, l, i}=P_{T x_{\eta}} \psi_{i}\left(r_{\eta, l}\right)^{-m}
$$

where $P_{T x_{\eta}}$ is the transmitted power, $\psi_{i}$ is the instantaneous value of the fading channel and shadowing gain, $r_{\eta, l}$ is the distance between the $i$-th transmitter and $N_{T x}$, and $m$ denotes the path-loss coefficient. The values $\psi_{i}$ and $r_{\eta, l}$ represent instantaneous values of the RVs $\Psi_{i}$ and $R_{\eta, l}$, respectively. $\Psi_{i}$ represents small-scale fading and shadowing effects. Small-scale fading amplitude is assumed to be Rayleigh distributed with mean power $2 \sigma_{\zeta}^{2}=1$. Lognormal shadowing is also assumed, with mean and standard deviation of the RV's natural logarithm given by $\mu_{\xi}=-\sigma_{\xi}^{2} / 2$ and $\sigma_{\xi}>0$, respectively. To simplify the composite fading model, we consider that the power of the Rayleigh and Lognormal effects can be jointly approximated by a Gamma distribution [10] with scale and shape parameters given by $\theta_{\psi_{i}}=\left(\frac{2(\vartheta+1)}{\vartheta}-1\right) \omega_{s}$ and $k_{\psi_{i}}=\frac{1}{\frac{2(\vartheta+1)}{\vartheta}-1}$, respectively, in which $\vartheta=\left(e^{\sigma_{\xi}^{2}}-1\right)^{-1}$ and $\omega_{s}=e^{\mu_{\xi}} \sqrt{\frac{\vartheta+1}{\vartheta}}$. 


\section{Wireless-Powered Communications}

We consider a WPC network with a time-switching protocol. In particular, wireless energy transfer is assumed in the downlink (DL) band, where the node $N_{T x}$ accumulates energy from the transmitters of the $v$ different coexisting wireless networks (Fig. 1). The node $N_{T x}$ first harvests energy during the time interval $c T$ from the DL RF band, and then uses it to transmit data to $N_{R x}$ over the uplink (UL) band. The transmission lasts $(1-c) T$, where $T$ is the total duration of a time-switching cycle and $c$ represents the time splitting factor. We consider an unitary cycle duration, i.e., $T=1$.

A Rayleigh fading channel between the nodes $N_{T x}$ and $N_{R x}$ is considered, and the distance between the nodes is denoted as $d_{1}$. The transmission power for information transfer depends on the energy harvested in the DL band and is denoted by $P_{N_{T x}}$. Consequently, the signal received by $N_{R x}$ can be written as

$$
y_{N_{T x}}=\frac{1}{\sqrt{d_{1}{ }^{m}}} \sqrt{P_{N_{T x}}} h_{1} x_{c}+n_{d},
$$

where $h_{1}$ is the channel coefficient from the transmitter $N_{T x}$ to the receiver $N_{R x}, x_{c}$ is the normalized information signal transmitted by $N_{T x}$, and $n_{d}$ is the zero-mean additive white Gaussian noise (AWGN) at the receiver.

\section{HARVESTED ENERGY}

In this section, we derive the energy harvested by the node $N_{T x}$ from the $v$ coexisting networks. Specifically, we describe how the aggregate power received by $N_{T x}$ from all transmitters of the $v$ coexisting networks can be approximated by an $\alpha-\mu$ distribution. Then, the aggregate power is used to derive the energy harvested during the harvesting period $c T$.

The work in [11] has proved that the aggregate power received from multiple nodes located over a single circle of a homogenous Poisson network is distributed according to a Gamma distribution. More recently, the work in [9] showed that the aggregate power received at the center of a SCM from the nodes located in an annulus $l$ can be also approximated by a Gamma distribution, when path-loss, fast fading and shadowing effects are considered. In our work, it is assumed the SCM described in [9].

Consequently, using the method of the moments, the shape and the scale parameters of the Gamma distribution that characterize the aggregate power received by $N_{T X}$ are given by

$$
k_{\eta, l}=\mathrm{E}\left[I_{\eta, l}\right]^{2} / \operatorname{Var}\left[I_{\eta, l}\right],
$$




$$
\theta_{\eta, l}=\operatorname{Var}\left[I_{\eta, l}\right] / \mathrm{E}\left[I_{\eta, l}\right]
$$

where $\mathrm{E}\left[I_{\eta, l}\right]$ and $\operatorname{Var}\left[I_{\eta, l}\right]$ are the expectation and variance of the power received from the transmitters of the annulus $l$ of the network $\eta$, which are respectively given by [9]

$$
\mathrm{E}\left[I_{\eta, l}\right]=2 \pi \lambda_{\eta, l} \tau_{\eta} P_{T x_{\eta}} e^{\mu_{\xi}} \sqrt{e^{\sigma_{\xi}^{2}}}\left(\frac{\left(R_{\eta, l+1}\right)^{2-m}-\left(R_{\eta, l}\right)^{2-m}}{2-m}\right),
$$

and

$$
\begin{aligned}
\operatorname{Var}\left[I_{\eta, l}\right]= & \pi \lambda_{\eta, l} \tau_{\eta} P_{T x_{\eta}}^{2} k_{\psi} \theta_{\psi}^{2}\left(1+k_{\psi}\right) \\
& \times\left(\frac{\left(R_{\eta, l+1}\right)^{2-2 m}-\left(R_{\eta, l}\right)^{2-2 m}}{1-m}\right) .
\end{aligned}
$$

To derive the aggregate power received from all nodes of a given network, $\Upsilon_{\eta}$, the summation of the power received from the $L_{\eta}$ annuli must be considered. Let $\left\{Z_{\eta, l}\right\}_{l=1}^{L_{\eta}}$ be independent non-identically distributed (i.n.i.d.) Gamma RVs with parameters $k_{\eta, l}$ and $\theta_{\eta, l}$. The aggregate power received from the network $\Upsilon_{\eta}$ can be written as $I_{\eta}=\sum_{l=1}^{L_{\eta}} Z_{\eta, l}$. In the same way, the aggregate power received by all coexisting network is written as $I_{a g g}=\sum_{\eta=1}^{v} I_{\eta}$, and represents the aggregate power caused by the nodes located within the $L_{N}$ annuli of the $v$ coexisting networks, with $L_{N}=v L_{\eta}$.

Let $\left\{Z_{j}\right\}_{j=1}^{L_{N}}$ be i.n.i.d. Gamma RVs with parameters $k_{j}$ and $\theta_{j}$, and $W_{j} \sim \operatorname{Nakagami}\left(m_{j}, \Omega_{j}\right)$. The aggregate power can be written as $I_{a g g}=\sum_{j=1}^{L_{N}} Z_{j}=\sum_{j=1}^{L_{N}} W_{j}^{2}$, since by definition $Z_{j}=W_{j}^{2}$, with $k_{j}=m_{j}$ and $\theta_{j}=\Omega_{j} / m_{j}$. According to [12], the sum of i.n.i.d. Nakagami- $m$ RVs can be accurately approximated by an $\alpha-\mu$ distribution. Consequently, the PDF of the aggregate power received from all transmitters of the coexisting networks $\left(I_{a g g}\right)$ can be approximated by an $\alpha-\mu$ distribution as follows

$$
f_{I_{a g g}}(y) \approx \frac{\alpha \mu^{\mu} y^{\alpha \mu-1}}{\hat{r}^{\alpha \mu} \Gamma(\mu)} \exp \left(-\mu \frac{y^{\alpha}}{\hat{r}^{\alpha}}\right)
$$

where $\hat{r}=\sqrt[\alpha]{\mathrm{E}\left[Y^{\alpha}\right]}$, and $\mu=\frac{\hat{r}^{2 \alpha}}{\operatorname{Var}\left[Y^{\alpha}\right]}$. To compute $f_{I_{a g g}}(y)$, the moment-based estimators for $\alpha$ and $\mu$ can be obtained from [12] as

$$
\frac{\Gamma^{2}(\mu+1 / \alpha)}{\Gamma(\mu) \Gamma(\mu+2 / \alpha)-\Gamma^{2}(\mu+1 / \alpha)}=\frac{\mathrm{E}^{2}\left[I_{a g g}\right]}{\mathrm{E}\left[I_{a g g}{ }^{2}\right]-\mathrm{E}^{2}\left[I_{\text {agg }}\right]},
$$

and

$$
\frac{\Gamma^{2}(\mu+2 / \alpha)}{\Gamma(\mu) \Gamma(\mu+4 / \alpha)-\Gamma^{2}(\mu+2 / \alpha)}=\frac{\mathrm{E}^{2}\left[I_{a g g}{ }^{2}\right]}{\mathrm{E}\left[{I_{a g g}}^{4}\right]-\mathrm{E}^{2}\left[{I_{a g g}}^{2}\right]} .
$$


The moments $\mathrm{E}\left[I_{a g g}\right], \mathrm{E}\left[{I_{a g g}}^{2}\right], \mathrm{E}\left[I_{a g g}{ }^{4}\right]$ in (5) and (6), can be computed using the following multinomial expansion

$$
\begin{aligned}
\mathrm{E}\left[I_{a g g}{ }^{n}\right] & =\sum_{n_{1}=0}^{n} \sum_{n_{2}=0}^{n_{1}} \cdots \sum_{n_{L_{N}-1}=0}^{n_{L_{N}-2}}\left(\begin{array}{c}
n \\
n_{1}
\end{array}\right)\left(\begin{array}{l}
n_{1} \\
n_{2}
\end{array}\right) \cdots\left(\begin{array}{l}
n_{L_{N}-2} \\
n_{L_{N}-1}
\end{array}\right) \\
& \times \mathrm{E}\left[W_{1}^{2\left(n-n_{1}\right)}\right] \mathrm{E}\left[W_{2}^{2\left(n_{1}-n_{2}\right)}\right] \cdots \mathrm{E}\left[W_{L_{N}}{ }^{2\left(n_{L_{N}-1}\right)}\right],
\end{aligned}
$$

where the Nakagami- $m$ moments are given as

$$
\mathrm{E}\left[W_{j}^{n}\right]=\frac{\Gamma\left(k_{j}+n / 2\right)}{\Gamma\left(k_{j}\right)}\left(\theta_{j}\right)^{n / 2} .
$$

Using the parameters $\alpha$ and $\mu$, the parameter $\hat{r}$ can be estimated by

$$
\hat{r}=\left[\frac{\mu^{2 / \alpha} \Gamma(\mu) \mathrm{E}\left[I_{a g g}\right]}{\Gamma(\mu+2 / \alpha)}\right]^{1 / 2} .
$$

Therefore, the harvested energy at the node $N_{T x}, E_{h}$, is written as

$$
E_{h}=\varsigma c T I_{a g g}
$$

where $0<\varsigma<1$ represents the energy conversion efficiency, and the RV $I_{\text {agg }}$ follows an $\alpha-\mu$ distribution with $\alpha, \mu$ and $\hat{r}$ computed from (5), (6), and (7).

\section{Throughrut AnALYsis}

After having harvested energy during the harvesting period $c T$, the node $N_{T x}$ transmits data in the UL band with $P_{N_{T x}}$ power, represented by

$$
P_{N_{T x}}=\frac{E_{h}}{(1-c) T}=\varsigma I_{a g g} \frac{c}{(1-c)} .
$$

Using (1), the signal-to-noise ratio (SNR) at the receiver node can be defined as

$$
\gamma_{d}=\frac{P_{N_{T x}}\left|h_{1}\right|^{2}}{d_{1}{ }^{m} \sigma_{n_{d}}^{2}}
$$

where $\sigma_{n_{d}}^{2}$ is the variance of the zero-mean AWGN. Considering a Rayleigh channel with mean power $2 \sigma_{h}^{2}$ between the nodes $N_{T x}$ and $N_{R x},\left|h_{1}\right|^{2}$ is exponentially distributed with parameter $1 /\left(2 \sigma_{h}^{2}\right)$. Given a SNR threshold $\gamma_{0}$, the outage probability of the transmission can be written as

$$
P_{\text {out }}\left(\gamma_{0}\right)=\mathrm{P}\left(\gamma_{d}<\gamma_{0}\right)=\int_{0}^{\gamma_{0}} f_{\gamma_{d}}(z) d z
$$

Using (10), and considering the PDFs of $I_{a g g}$ and $\left|h_{1}\right|^{2}$, (11) can be rewritten as

$$
P_{\text {out }}\left(\gamma_{0}\right)=\frac{\alpha \mu^{\mu}}{\hat{r}^{\alpha \mu} \Gamma(\mu)} \frac{1}{\Lambda} \int_{0}^{\gamma_{0}} \int_{0}^{\infty} \frac{1}{y}\left(\frac{z}{y}\right)^{\alpha \mu-1} \exp \left(\frac{-\mu z^{\alpha}}{(\hat{r} y)^{\alpha}}-\frac{1}{2 \sigma_{h}^{2} \Lambda} y\right) d y d z
$$


where

$$
\Lambda=\frac{\varsigma c}{(1-c) d_{1}{ }^{m} \sigma_{n_{d}}^{2}},
$$

is the scaling value of the product of the RVs $I_{a g g}$ and $\left|h_{1}\right|^{2}$. Since the integral in (12) can only be numerically solved, we propose to approximate $P_{\text {out }}\left(\gamma_{0}\right)$ by an $\alpha$ - $\mu$ distribution as follows

$$
P_{\text {out }}\left(\gamma_{0}\right) \approx \Gamma\left(\mu_{p}, \mu_{p}\left(\frac{\gamma_{0}}{\hat{r}_{p} \Lambda}\right)^{\alpha_{p}}\right) \frac{1}{\Gamma\left(\mu_{p}\right)},
$$

where $\alpha_{p}, \mu_{p}$ and $\hat{r}_{p}$ can be obtained by solving the system of equations formed by (5), (6) and (7), substituting the symbols $\alpha, \mu, \hat{r}$, and $\mathrm{E}\left[{I_{a g g}}^{n}\right]$ by $\alpha_{p}, \mu_{p}, \hat{r}_{p}$, and

$$
\mathrm{E}\left[\left(I_{a g g}\left|h_{1}\right|^{2}\right)^{n}\right]=n !\left(2 \sigma_{h}^{2} \hat{r}\right)^{n} \frac{\Gamma(\mu+n / \alpha)}{\mu^{n / \alpha} \Gamma(\mu)},
$$

respectively. As will be seen, (13) shows to be a very tight approximation, being evaluated instantaneously. (14) represents the $n$-th moment of the product of the RVs $I_{a g g}$ and $\left|h_{1}\right|^{2}$. Assuming a communication rate $\mathcal{R}$ (in bits/T) and the transmission duration $(1-c) T$, the throughput of the communication channel between $N_{T x}$ and $N_{R x}$ can be written as

$$
R_{\tau}(c)=\left(1-P_{\text {out }}\left(\gamma_{0}\right)\right)(1-c) \mathcal{R}
$$

From (15), one can notice that $\left(1-P_{\text {out }}\left(\gamma_{0}\right)\right)$ increases with $c$. However, the transmission duration decreases with $c$. This fact has motivated us to derive the optimal time allocation ratio,

$$
c^{*}=\arg \max _{0<c<1} R_{\tau}(c),
$$

which can only be computed in a numerical way, by finding the value $c^{*}$ for which the following condition holds

$\alpha_{p}\left(c^{*} \Gamma\left(\mu_{p}\right)\right)^{-1} \exp \left(\mu_{p}\left(\frac{\gamma_{0} d_{1}^{m} \sigma_{n_{d}}^{2}}{\hat{r}_{p} \varsigma} \frac{1-c^{*}}{c^{*}}\right)^{\alpha_{p}}\right)=1-\left(\Gamma\left(\mu_{p}\right)\right)^{-1} \Gamma\left(\mu_{p}, \mu_{p}\left(\frac{\gamma_{0} d_{1}{ }^{m} \sigma_{n_{d}}^{2}}{\hat{r}_{p} \varsigma} \frac{1-c^{*}}{c^{*}}\right)^{\alpha_{p}}\right)$.

\section{Model VAlidation AND Discussions}

In this section, we validate the methodology described in Sections III and IV, by comparing the numerical results with simulations. We consider two coexisting networks $(v=2)$ : a static network $\left(\Upsilon_{1}\right)$, and a network $\left(\Upsilon_{2}\right)$ where the nodes move according to the RWP. Three different mobility scenarios are analysed for $\Upsilon_{2}$, considering the case where nodes are static $(\mathrm{E}[V]=0$ $\mathrm{m} / \mathrm{s})$, or mobile with different average velocities $(\mathrm{E}[V]=\{10.82,1.50\} \mathrm{m} / \mathrm{s})$. The parameters adopted in the validation are presented in Table I, which are divided in the parameters related with 
the "Propagation Effects" described in Section II-B, the parameters of the network $\Upsilon_{1}$ ("Static Network"), the parameters of the network $\Upsilon_{2}$ ("Mobile Network"), and other parameters adopted in the WPC model (Section II-C), SCM, and simulations. The assessment of the model is carried out by comparing Monte Carlo simulation results with numerical results of the aggregate power ( $I_{a g g}$ approximated by (4)), the throughput $\left(R_{\tau}(c)\right.$ computed with (15)), and the optimal time allocation ratio $\left(c^{*}\right.$ computed with (17)). In the SCM, we have considered the same circular area for both networks $\Upsilon_{1}$ and $\Upsilon_{2}$, i.e., $R_{1,1}=R_{2,1}=20 \mathrm{~m}, R_{1, L_{\eta}+1}=R_{2, L_{\eta}+1}=120 \mathrm{~m}$, and $\rho_{1}=\rho_{2}=20 \mathrm{~m}$.

In Fig. 2, we compare the cumulative distribution function (CDF) of the aggregate power $\left(I_{\text {agg }}\right)$ generated by the coexisting networks $\left(\Upsilon_{1}\right.$ and $\left.\Upsilon_{2}\right)$ for the different mobility scenarios considered in network $\Upsilon_{2}(E[V]=\{10.82 \mathrm{~m} / \mathrm{s}, 1.50 \mathrm{~m} / \mathrm{s}, 0 \mathrm{~m} / \mathrm{s}\})$. Table II presents the $\alpha-\mu$

TABLE I

PARAMETERS ADOPTED IN THE SIMULATIONS.

\begin{tabular}{|c|c|c|c|c|}
\hline \multirow{4}{*}{$\begin{array}{c}\text { Propagation } \\
\text { Effects }\end{array}$} & $P_{T x_{\eta}}$ & $10^{3} \mathrm{~mW}$ & $m$ & 2 \\
\cline { 2 - 5 } & $\sigma_{\xi}$ & 0.69 & $\sigma_{h}{ }^{2}$ & $1 / 2$ \\
\cline { 2 - 4 } & $\sigma_{n_{d}}^{2}$ & $0.01 \mathrm{~mW}$ & \multicolumn{2}{|c}{} \\
\hline
\end{tabular}

\begin{tabular}{|c|c|c|c|c|}
\hline \multirow{2}{*}{$\begin{array}{c}\text { Static } \\
\text { Network } \\
\left(\Upsilon_{1}\right)\end{array}$} & $R_{1,1}$ & $20 \mathrm{~m}$ & $R_{1, L_{\eta}+1}$ & $120 \mathrm{~m}$ \\
\cline { 2 - 5 } & $\rho_{1}$ & $20 \mathrm{~m}$ & $L_{1}$ & 5 \\
\cline { 2 - 5 } & $\lambda_{1}\left(\right.$ node $\left./ \mathrm{m}^{2}\right)$ & $1 \times 10^{-4}$ & \multicolumn{3}{|c}{} \\
\hline
\end{tabular}

\begin{tabular}{|c|c|c|c|c|}
\hline \multirow{4}{*}{$\begin{array}{c}\text { Mobile } \\
\text { Network } \\
\left(\Upsilon_{2}\right)\end{array}$} & $R_{2,1}$ & $20 \mathrm{~m}$ & $R_{2, L_{\eta}+1}$ & $120 \mathrm{~m}$ \\
\cline { 2 - 5 } & $\rho_{2}$ & $20 \mathrm{~m}$ & $L_{2}$ & 5 \\
\cline { 2 - 5 } & $V_{\min }$ & $5 \mathrm{~m} / \mathrm{s}$ & $V_{\max }$ & $20 \mathrm{~m} / \mathrm{s}$ \\
\cline { 2 - 5 } & $n_{2}$ & 100 & $\tau_{2}$ & 1 \\
\cline { 2 - 5 } & $\mathrm{E}[V]$ & $\{10.82,1.50,0\} \mathrm{m} / \mathrm{s}$ & $X_{\max }$ & $1000 \mathrm{~m}$ \\
\cline { 2 - 5 } & $T_{p}$ & $\{0,300,3000\} \mathrm{s}$ & $Y_{\max }$ & $1000 \mathrm{~m}$ \\
\hline
\end{tabular}

\begin{tabular}{|c|c|c|c|c|}
\hline \multirow{2}{*}{ Others } & $\begin{array}{c}\text { Monte Carlo } \\
\text { samples }\end{array}$ & $3 \times 10^{6}$ & $d_{1}$ & $5 \mathrm{~m}$ \\
\cline { 2 - 5 } & $\gamma_{0}$ & $\{-10,-5,0,5,10,15\} \mathrm{dB}$ & $\varsigma$ & 1 \\
\cline { 2 - 5 } & $L_{N}$ & 10 & $v$ & 2 \\
\hline
\end{tabular}


distribution parameters adopted in (4) to approximate $I_{a g g}$ for the different average velocities $\mathrm{E}[V]$. As can be seen, the numerical results (represented by the "Model" curves) are close to the results obtained through simulation. This indicates that the $\alpha-\mu$ distribution in (4) can effectively approximate the distribution of $I_{a g g}$ with high accuracy. Moreover, we observe that the aggregate power increases with the mobility of the network $\Upsilon_{2}$, which is due to the higher density of nodes closer located to $N_{T x}$ as the node's mobility increase [7].

TABLE II

PARAMETERS ESTIMATED FOR THE MODEL CURVES OF $I_{a g g}$.

\begin{tabular}{|c|c|c|c|c|}
\cline { 2 - 5 } \multicolumn{2}{c|}{} & $\alpha$ & $\mu$ & $\hat{r}$ \\
\hline \multirow{3}{*}{ Fig. 2} & $\mathrm{E}[V]=10.82 \mathrm{~m} / \mathrm{s}$ & 0.198 & 49.032 & 2.946 \\
\cline { 2 - 5 } & $\mathrm{E}[V]=1.50 \mathrm{~m} / \mathrm{s}$ & 0.263 & 19.548 & 1.881 \\
\cline { 2 - 5 } & $\mathrm{E}[V]=0 \mathrm{~m} / \mathrm{s}$ & 0.275 & 16.621 & 1.714 \\
\hline
\end{tabular}

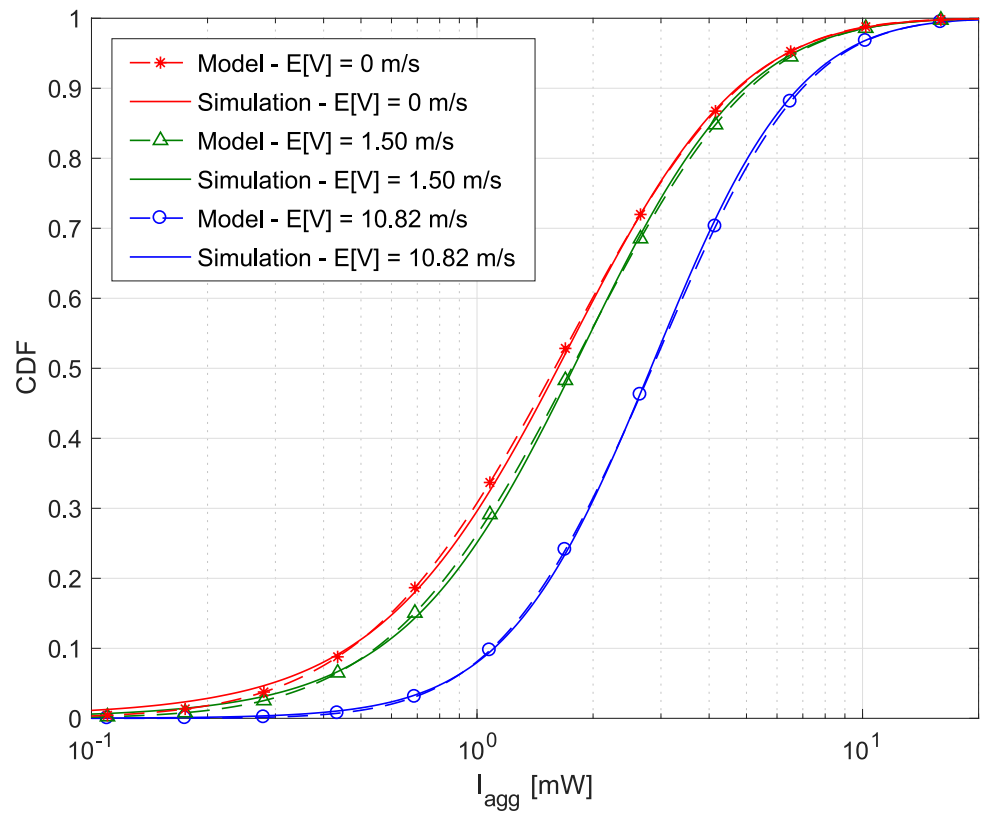

Fig. 2. CDF of the aggregate power when $\Upsilon_{1}$ and $\Upsilon_{2}$ coexist, and considering different average speeds of $\Upsilon_{2}$ 's nodes.

Next, we characterize the throughput $\left(R_{\tau}\right)$ achieved by the WPC system in the same coexisting scenarios adopted to obtain the results illustrated in Fig. 2. To this purpose, we have considered the optimal energy conversion efficiency of the energy harvesting process $(\varsigma=1)$, 
the communication range $d_{1}=5 \mathrm{~m}$, and the SNR threshold $\gamma_{0}=5 \mathrm{~dB}$ at the receiver. Moreover, it is assumed that $N_{T x}$ uses all the harvested energy to transmit the information. In Fig. 3, we present different curves of the throughput as a function of the time splitting ratio $c$. The numerical results (represented by the "Model" curves) have considered the approximation proposed in (13) to compute the outage probability. The parameters used in (13) were previously computed as described in Section IV, and their values for the different mobility scenarios are presented in Table III. The throughput, computed with (15), is close to the throughput obtained in the Monte Carlo simulations, as illustrated in Fig. 3. The results show that higher throughput values are achieved for higher mobility scenarios of the RWP network $\Upsilon_{2}$. This is because the amount of harvest energy increases with the velocity of the nodes of the network $\Upsilon_{2}$, as justified by the aggregate power results in Fig. 2. Moreover, it is shown that the parameter $c$ effectively impacts on the achieved throughput, clearly identifying an upward where extending the harvesting period increases the transmission power, and a downward zone, where the extension of the harvesting period shortens the transmission period. The optimal point of operation is the one that divide the two zones.

TABLE III

Parameters estimated for the Model curves of $P_{\text {out }}$.

\begin{tabular}{|c|c|c|c|c|}
\cline { 3 - 5 } \multicolumn{2}{c|}{} & $\alpha_{p}$ & $\mu_{p}$ & $\hat{r}_{p}$ \\
\hline \multirow{3}{*}{ Fig. 3 } & $\mathrm{E}[V]=10.82 \mathrm{~m} / \mathrm{s}$ & 0.316 & 6.098 & 2.199 \\
\cline { 2 - 5 } & $\mathrm{E}[V]=1.50 \mathrm{~m} / \mathrm{s}$ & 0.287 & 6.350 & 1.347 \\
\cline { 2 - 5 } & $\mathrm{E}[V]=0 \mathrm{~m} / \mathrm{s}$ & 0.284 & 6.288 & 1.216 \\
\hline
\end{tabular}

To evaluate the optimal time allocation ratio $\left(c^{*}\right)$ proposed in (17), we have considered the higher mobility scenario $(E[V]=10.82 \mathrm{~m} / \mathrm{s})$, and multiple SNR thresholds $\left(\gamma_{0}=\{-10,-5,0,5,10,15\}\right.$ $\mathrm{dB})$. The simulation results of the throughput and the numerical results of the optimal time allocation ratio $\left(c^{*}\right)$ are illustrated in Fig. 4 (a marker "o" was adopted to indicate the $c^{*}$ value numerically computed with (17)). The throughput inversely increases with $\gamma_{0}$, as expected. We also observe that $\gamma_{0}$ also impacts on the shape of the throughput curves. However, the optimal time allocation ratio is accurately approximated by (17) for all $\gamma_{0}$ values, as depicted in the figure. 


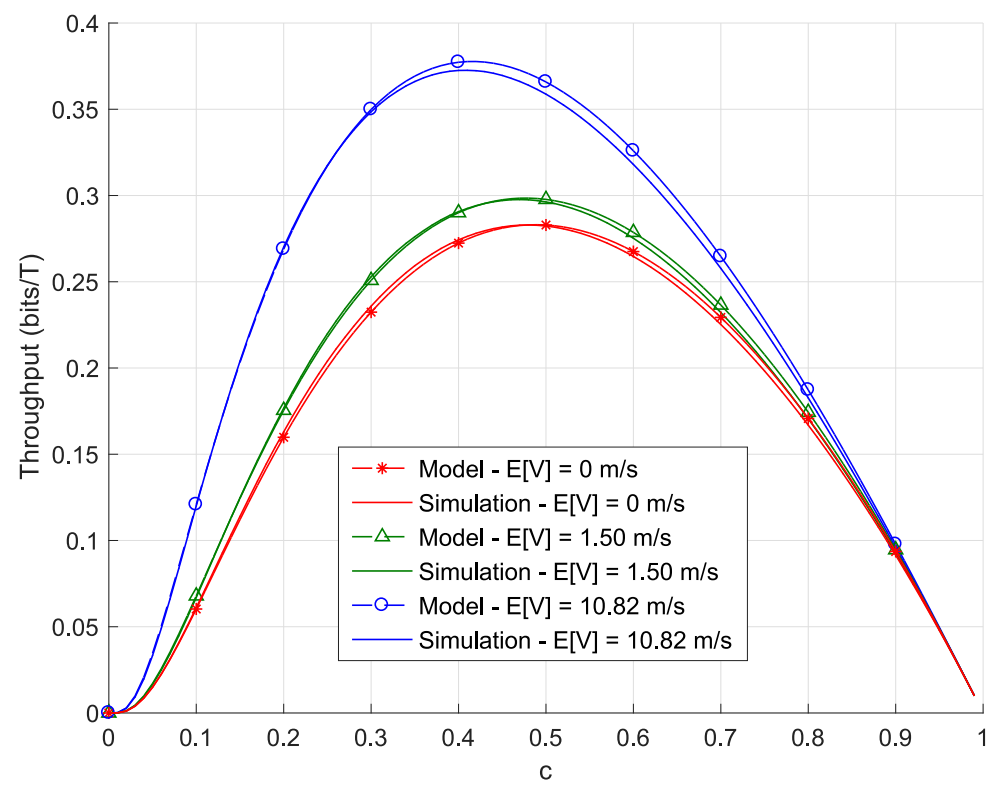

Fig. 3. Throughput $R_{\tau}$ for the scenario considered in Fig. 2.

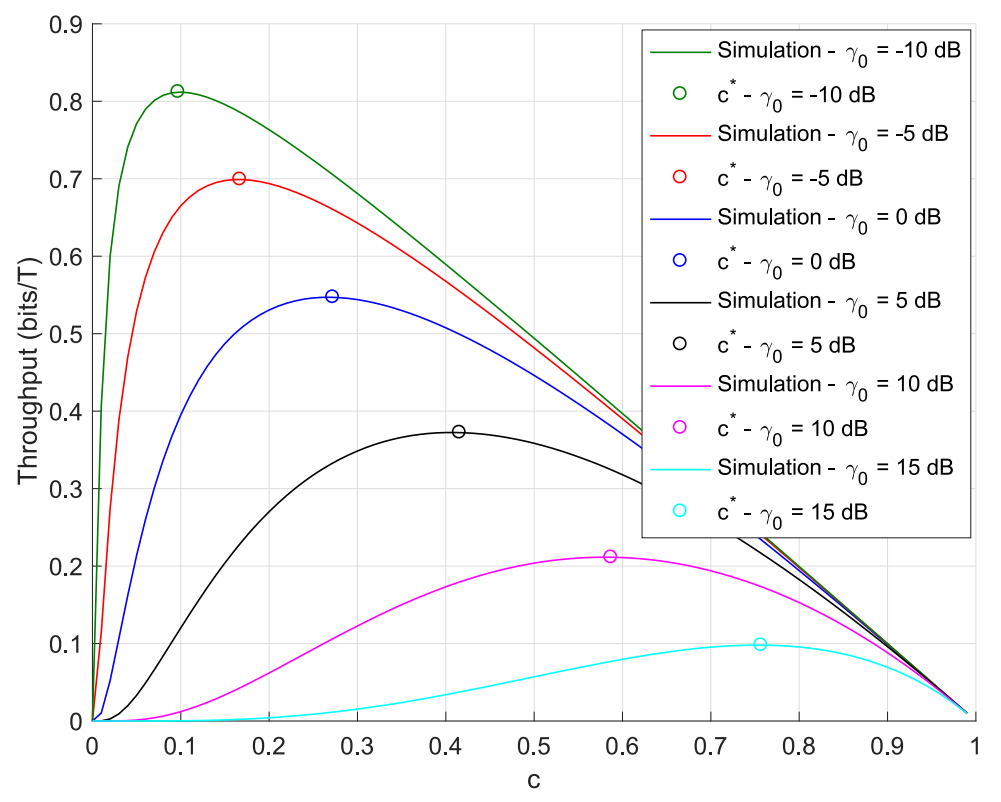

Fig. 4. Optimal time allocation ratio $c^{*}$ for different SNR thresholds $\gamma_{0}$. 


\section{REFERENCES}

[1] L. Irio, R. Oliveira, and D. B. da Costa, "Highly accurate approaches for the interference modeling in coexisting wireless networks," IEEE Commun. Lett., pp. 1-1, 2019.

[2] Y. Gu and S. Aïssa, "RF-based energy harvesting in decode-and-forward relaying systems: Ergodic and outage capacities," IEEE Trans. Wireless Commun., vol. 14, no. 11, pp. 6425-6434, Nov. 2015.

[3] Y. Chen, "Energy-harvesting AF relaying in the presence of interference and Nakagami-m fading," IEEE Trans. Wireless Commun., vol. 15, no. 2, pp. 1008-1017, Feb. 2016.

[4] I. Flint, X. Lu, N. Privault, D. Niyato, and P. Wang, "Performance analysis of ambient RF energy harvesting with repulsive point process modeling," IEEE Trans. Wireless Commun., vol. 14, no. 10, pp. 5402-5416, Oct. 2015.

[5] Y. L. Che, L. Duan, and R. Zhang, "Spatial throughput maximization of wireless powered communication networks," IEEE J. Sel. Areas Commun., vol. 33, no. 8, pp. 1534-1548, Aug. 2015.

[6] I. S. Gradshteyn and I. M. Ryzhik, Table of Integrals, Series, and Products, 7th ed. New York, NY, USA: Academic Press, 2007.

[7] C. Bettstetter, G. Resta, and P. Santi, "The node distribution of the random waypoint mobility model for wireless ad hoc networks," IEEE Trans. Mobile Comput., vol. 2, no. 3, pp. 257-269, Jul. 2003.

[8] L. Irio, R. Oliveira, and L. Bernardo, "Aggregate interference in random waypoint mobile networks," IEEE Commun. Lett., vol. 19, no. 6, pp. 1021-1024, Jun. 2015.

[9] L. Irio, A. Furtado, R. Oliveira, L. Bernardo, and R. Dinis, "Interference characterization in random waypoint mobile networks," IEEE Trans. Wireless Commun., vol. 17, no. 11, pp. 7340-7351, Nov. 2018.

[10] S. Al-Ahmadi and H. Yanikomeroglu, "On the approximation of the Generalized-K distribution by a Gamma distribution for modeling composite fading channels," IEEE Trans. Wireless Commun., vol. 9, no. 2, pp. 706-713, Feb. 2010.

[11] M. Haenggi and R. K. Ganti, "Interference in large wireless networks," Found. Trends Netw., vol. 3, no. 2, pp. 127-248, Nov. 2009.

[12] D. B. da Costa, M. D. Yacoub, and J. C. S. Santos Filho, "An improved closed-form approximation to the sum of arbitrary Nakagami-m variates," IEEE Trans. Veh. Technol., vol. 57, no. 6, pp. 3854-3858, Nov. 2008. 